\title{
Effect of High Frequency Substrate Noise on LC-VCOs
}

\author{
Marc Molina, Xavier Aragones, Diego Mateo, José Luis González \\ Electronic Engineering Department \\ Universitat Politècnica de Catalunya \\ Barcelona, Spain
}

\begin{abstract}
This paper presents an experimental analysis of the performance degradation of an LC-Voltage Controlled Oscillator (LC-VCO) produced by high frequency noise present in the substrate. The spurs observed are shown to be caused by a frequency pulling mechanism. Based on the theory of injection locked oscillators, a new analytical model to predict the behavior of the LC-VCO under the effect of high frequency substrate noise is presented. The analytical model, which is successfully compared with experimental measurements on a $7 \mathrm{GHz}$ LCVCO, provides rapid intuition on the relation between spurs and circuit parameters.
\end{abstract}

\section{INTRODUCTION}

The single chip integration of an RF communication system faces a lot of challenges and problems due to the undesired interaction between its blocks, such as the harmful interferences that digital circuitry produces over a delicate RF or microwave front end. In particular, substrate noise can affect the Voltage Controlled Oscillator (VCO) performance in terms of output spectral purity, phase noise and it can even modify the oscillation frequency. Digital circuitry is not the only possible origin of substrate noise, but other RF blocks of the same transceiver can interfere with the VCO as well. One example is the Power Amplifier which will normally inject noise at a frequency very close to the VCO fundamental output frequency.

The effect of low frequency substrate noise on a VCO has been widely studied [1], [2], determining both the coupling mechanisms and the performance degradation. The effect when the substrate noise frequency is close to the VCO output frequency presents important differences with respect to the low-frequency case, both in the coupling mechanisms and in the performance degradation. Consequently, new design guidelines should be stated to protect the LC-VCOs from high frequency substrate noise.

This work presents an analytical model (and its experimental verification) of the VCO degradation due to a high frequency noise injected into the substrate, based on the injection locked oscillator theory. The presented model identifies which are the key factors that contribute to the LC-VCO performance degradation.

This works was partially funded by EU-Feder and Spanish MICINN under TEC2005-06784 and TEC2008-01856 projects and AGAUR SGR 1497 funds.

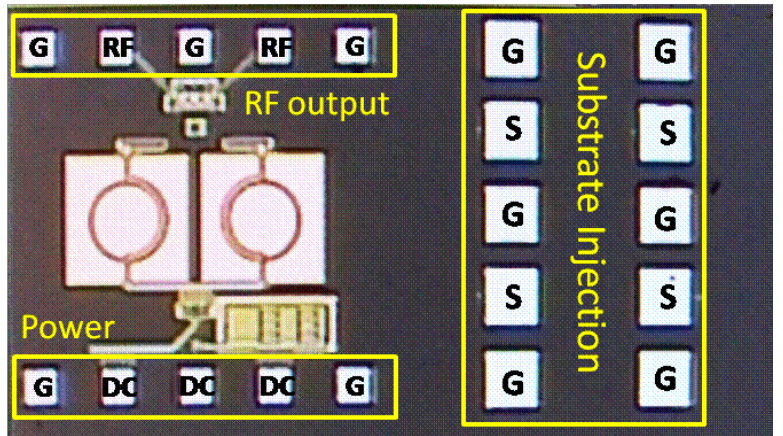

Fig. 1. Test chip microphotography

The LC-VCO and the measurement setup are presented in Section II. The experimental effect of the substrate noise on the VCO is shown in Section III. Section IV presents and analyzes the new analytical model. Finally, some measures to reduce the VCO performance degradation are proposed on Section V.

\section{TEST CHIP AND MEASUREMENT SETUP}

A $7 \mathrm{GHz}$ LC-VCO has been fabricated in a CMOS $0.18 \mu \mathrm{m}$ technology using a differential NMOS topology. The VCO has a power consumption of $15 \mathrm{~mW}$ from a $1.8 \mathrm{~V}$ supply voltage. Its output frequency sweeps from $6 \mathrm{GHz}$ to $8 \mathrm{GHz}$ with a maximum sensitivity to the control voltage of $2 \mathrm{GHz} / \mathrm{V}$.

Substrate noise injection pads have been included in the layout design in order to directly inject the noise signal into the substrate. Fig.1 shows a microphotography of the chip where the injection pads can be appreciated. The interfering noise is generated with a single tone signal generator, and it is injected in the substrate through a GSG RF probe contacting a pad that is capacitive coupled to the substrate. The RF outputs, DC and power pads have been wire bonded to a PCB.

\section{EXPERIMENTAL ANALYSIS}

The injection into the substrate of a sinusoidal tone with a frequency close to the fundamental output frequency of the VCO, $\omega_{o}$, generates two symmetric sideband spurs, one at the injected frequency, $\omega_{i}$, and another one at $2 \omega_{o}-\omega_{i}$. Fig.2 shows a typical observation of this effect. As the frequency offset is increased, the amplitude of the spurs falls at a rate of $20 \mathrm{~dB} / \mathrm{dec}$, as shown in Fig.3. An important characteristic of 


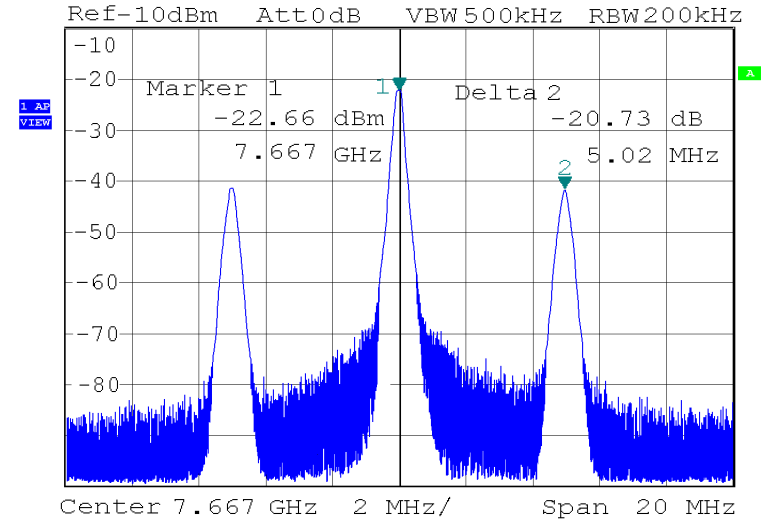

Fig. 2. Output spectrum of the VCO perturbed by a substrate coupled noise at a $5 \mathrm{MHz}$ frequency offset from the carrier

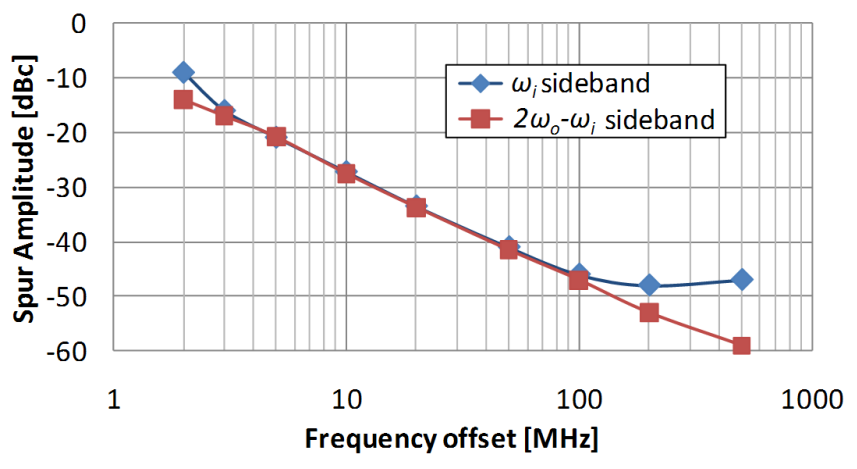

Fig. 3. Spur amplitude for different frequency offsets

the sideband spurs is that they have approximately the same amplitude except for very high offsets, where the spur at $\omega_{i}$ saturates. This characteristic has been confirmed for different VCO frequencies and different injected power.

A possible explanation of the generated spurs, especially the one at $2 \omega_{o}-\omega_{i}$, has been previously proposed in the literature [3], [4] as an intermodulation between the VCO output and the injected tone due to the nonlinearities of the resonant tank. Nevertheless, the effect of intermodulation cannot explain the observed characteristics of the sideband spurs. Particularly, assuming intermodulation, the amplitude of the generated spur at $2 \omega_{o}-\omega_{i}$ should depend on the power of the VCO output and of the injected signal [3], while the amplitude of the spur at $\omega_{i}$ should only depend on the injected power. On the other hand, both amplitudes shouldn't depend on the frequency offset.

An alternative explanation for the spurs is proposed in this paper. The problem is analyzed from the perspective of an injection locked oscillator (ILO), where the injected signal is small and not too close to the VCO output. Under these circumstances, the injected signal cannot lock the VCO but is enough to perturb its output spectrum.

Back in 1946, Adler [5] described and formulated the behavior of locked and unlocked ILOs. Subsequently, his

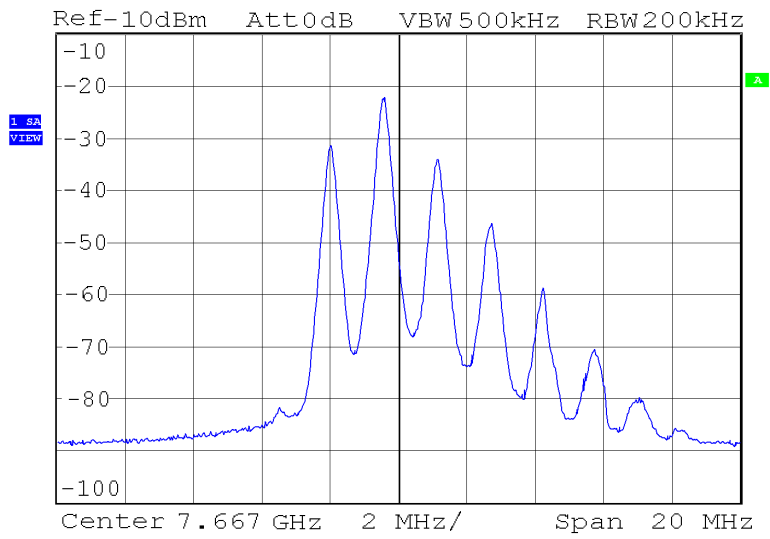

Fig. 4. Output spectrum of the quasi-locked VCO

work was extended by Stover and Armand [6], [7] and more recently by Razavi [8]. As described in these works, the injected signal can modify the instantaneous phase of the oscillator, and consequently, its instantaneous frequency. In the particular case where the injected signal is small compared to the oscillator signal or its frequency is relatively far from it, the output of the oscillator is modulated in frequency due to the frequency pulling effect [6]. The output spectrum of the oscillator under these circumstances presents two symmetric sidebands with the same amplitude separated from the VCO fundamental $\omega_{o}$ by an offset equal to $\omega_{o}-\omega_{i}$, exactly as observed in Fig.2.

As the frequency of the injected signal gets closer to the VCO output or its power is increased, the pulling effect becomes stronger. The VCO frequency is slightly shifted towards the injected signal and several sidebands appear in its opposite side. In this situation, a slight reduction of the offset or a small increase on the injected power will force the VCO to lock to the injected signal.

In order to confirm that the high frequency substrate noise can force the VCO to behave that way, the power of the injected noise used in the experiment of Fig.2 is increased and the frequency offset is gradually reduced. Fig.4 shows the output spectrum of the VCO when a $15 \mathrm{dBm}$ noise is injected in the substrate with an offset of $2 \mathrm{MHz}$ from the VCO frequency. The output frequency of the VCO has been pulled around $0.5 \mathrm{MHz}$ towards the noise signal, which is the only spur on the left side of the VCO signal. On the right side, several spurs have been generated by the pulling effect. As expected, if the frequency offset is further reduced, the VCO gets locked to the noise tone. Fig.5 shows the measured locking range for different injected noise power levels. Right vertical axis shows the estimated noise power at the tank.

\section{ANALYTICAL MODEL}

The output voltage of an oscillator affected by an externally injected signal is described by the following equation:

$$
v(t)=A(t) \sin \left[\omega_{i} t+\varphi(t)\right]
$$




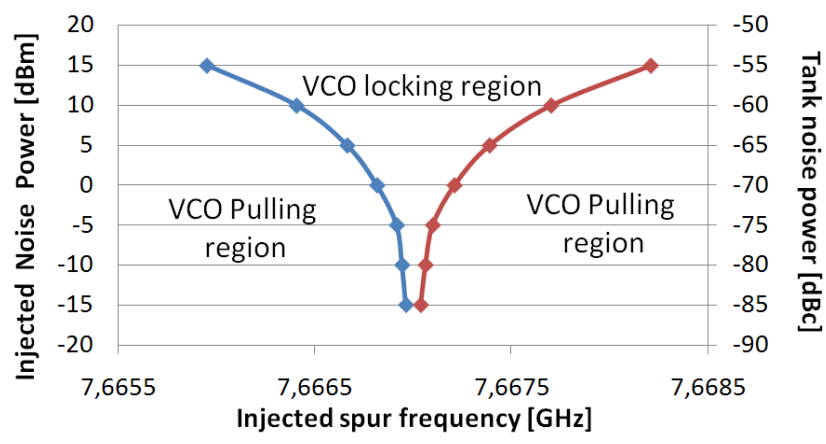

Fig. 5. Measured locking range for different substrate noise power

where $\omega_{i}$ is the frequency of the noise injected signal, $A(t)$ is the output amplitude and $\varphi(t)$ is the differential phase between the injected signal and the VCO output.

Intuitively, the phase of the oscillator is perturbed by the injected signal. When locked, $\varphi(t)$ is constant and the VCO is forced to oscillate at the injected frequency, $\omega_{i}$. When the noise signal is too weak or too far from the free oscillation frequency, it does not succeed in pulling the oscillator into the locking state, the injected signal and the VCO have different frequencies and, consequently, the phase difference varies with time, modifying the instantaneous frequency and even the amplitude of the output signal.

Early work by Adler showed that the phase difference between both signals is described by the following differential equation [5]:

$$
\frac{\partial}{\partial t} \varphi(t)=B \sin [\varphi(t)]-\left(\omega_{i}-\omega_{o}\right)
$$

where,

$$
B=\frac{A_{i}}{A_{o}} \frac{\omega_{o}}{2 Q}
$$

being $A_{i}$ and $A_{o}$ the amplitudes of the perturbing noise signal coupled to the VCO tank and VCO signal respectively, and $Q$ the quality factor of the LC-VCO resonant tank. From a physical point of view, $B$ represents the maximum frequency offset, $\omega_{o}-\omega_{i}$, where locking is possible, i.e the locking range.

The differential equation (2) can be mathematically solved into a closed but complex and very little intuitive solution that has to be numerically solved or simplified for particular cases [6], [7], [9] in order to provide information about the oscillator behavior.

A simplification approach is followed in this work in order to provide an intuitive solution to (2), suitable to predict the amplitude and the origin of the two sidebands in the particular scenario where the noise couples to the VCO through the substrate. This scenario presents some particular characteristics that differ from the general case of ILOs theory, where the power of the injected tone is very high and the ILO is usually locked or about to lock. The power of the substrate noise signal reaching the tank is generally extremely small compared to the output power of the VCO, and consequently, from (3), the resulting locking range is very small. Under these circumstances, the weak pulling caused by the substrate noise can only slightly modify the center frequency of the VCO output. Based on the assumption that the sidebands are then caused by the frequency modulation of the VCO output, the following solution to the differential equation (2) is presented as a valid approximation to obtain the phase of the substrate noise perturbed VCO:

$$
\varphi(t)=\left(\omega_{o}-\omega_{i}\right) t-\frac{B}{\left(\omega_{o}-\omega_{i}\right)} \cos \left[\left(\omega_{o}-\omega_{i}\right) t\right]
$$

It can be easily proven that the accuracy of (4) in predicting the phase of the pulled VCO depends on the difference between the frequency offset and the locking range. The accuracy of (4) grows as the noise frequency gets far from the VCO center frequency, correctly predicting the frequency and amplitude of the sidebands.

The approximated time domain behavior of the VCO perturbed by substrate noise is obtained following (1), (3) and (4):

$v(t) \simeq A_{o} \sin \left[\omega_{o} t-\frac{A_{i}}{A_{o}} \frac{\omega_{o}}{2 Q\left(\omega_{o}-\omega_{i}\right)} \cos \left[\left(\omega_{o}-\omega_{i}\right) t\right]\right]$

This solution can be seen as a narrowband frequency modulation of a pure tone being $\omega_{o}$ the carrier frequency, $\omega_{o}-\omega_{i}$ the modulating frequency and $B /\left(\omega_{o}-\omega_{i}\right)$ the modulation index or frequency deviation. Consequently, the relative amplitude of the spectrum spurs can be obtained through the first order Bessel function:

$$
\begin{aligned}
\text { SpurRelAmplitude } & =J_{1}\left(\frac{A_{i}}{A_{o}} \frac{\omega_{o}}{2 Q\left(\omega_{o}-\omega_{i}\right)}\right) \\
& \simeq 0.5 \frac{A_{i}}{A_{o}} \frac{\omega_{o}}{2 Q\left(\omega_{o}-\omega_{i}\right)}
\end{aligned}
$$

The accuracy of (5) is verified by comparison against the measurements on the test chip described above. Fig.6 shows the measured relative amplitude of both sideband spurs compared against the amplitude predicted by the proposed analytical model (5) and with the exact mathematical solution to (2). Note that the amplitude of the injected signal that reaches the tank, $A_{i}$, should be obtained from simulation, from direct measurement of the tank signal with the VCO turned off or from (3) after measuring the locking range.

The proposed analytical model shows very good alignment with the exact solution of (2), accurately predicting the relative amplitude of the measured sidebands except for very small frequency offsets, where the VCO is extremely close to lock to the substrate noise signal. Beyond $100 \mathrm{MHz}$ the amplitude of the spur at $\omega_{i}$ saturates due to the direct coupling of the injected signal into the VCO tank and to the output buffers. Obviously, this effect is not represented by any of the pulling models.

\section{REDUCTION OF HIGH FREQUENCY SPURS}

The new analytical model just introduced presents a more particular, intuitive and simple solution than the general theory of unlocked ILO in order to analyze the effect of the high 

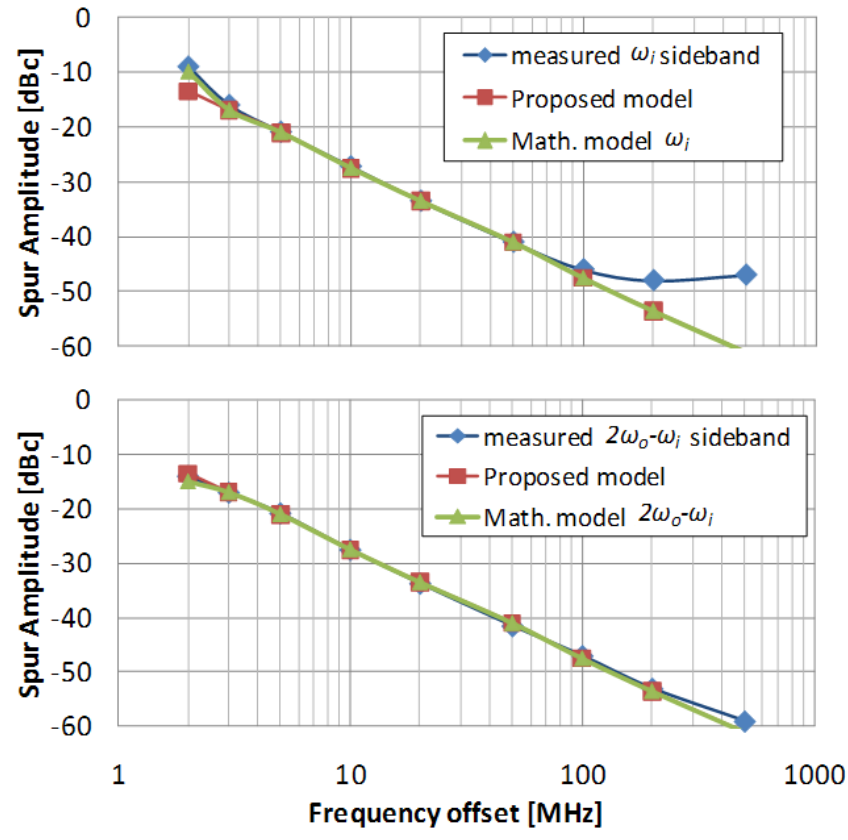

Fig. 6. Comparison between measurements and models

frequency substrate noise on LC-VCOs. It also presents a closed expression (6) that provides a rapid identification of the key factors that contribute to the LC-VCO performance degradation, providing directions on the measures that a designer can take to improve the robustness of a LC-VCO in front of high frequency substrate noise.

Observing expression (6), the spur amplitude only depends on the VCO and noise amplitudes, the tank quality factor and the VCO and offset frequencies. Optimizing these variables for spur minimization introduces several tradeoffs from the design point of view. The tank quality factor is usually determined by the inductor physical characteristics and tradesoff with the area and cost. The VCO output amplitude tradesoff with the power consumption and may be conditioned by the input requirements of other blocks of the system. Although these factors can already be optimized during design process of VCOs with stringent specifications, other situations may allow room to introduce spur minimization as a design criteria.

Beyond these factors related to the VCO circuit design, expression (6) highlights a third option which is reducing the amount of noise reaching the tank. The most appropriate measure to reduce the noise effect on each particular case will depend on the path that the noise follows to reach the tank, like for example, coupling to the VCO transistors, to the tank inductor or to the power and control lines (ground, Vdd, control voltage, current biasing, etc).

In our particular case, off-chip decoupling capacitors were included in the board that contains the circuit of Fig.1, proving to be effective to reduce the spurs amplitude by about $8 d B s$ and indicating that, in our test chip, power lines are a dominant coupling path between the substrate and the VCO.
The high efficiency of the decoupling capacitors at this range of frequencies suggests that, once the power lines become correctly decoupled, the noise coupling through the inductor and VCO transistors would be the main contributor to the spur amplitude. Protection structures, such as inductor shields or substrate guard rings, can then be used [4] to increase the isolation between the inductor and the substrate. The effectiveness of the protection structures at high frequency is highly limited by the impedance of the noise return path to ground [2], [10], thus, especial care should be taken when designing the connection of the protection structures to ground.

\section{CONCLUSION}

In this work it has been proven that a high frequency tone traveling through the substrate can seriously degrade the performance of an LC-VCO. The output frequency of the VCO is FM modulated by the pulling effect caused by the substrate noise, generating two symmetrical sidebands around the VCO signal in the output spectrum.

A simple analytical model to predict the amplitude of the sidebands has been presented and verified against the measurements. The model reveals the main contributors to the LC-VCO performance degradation helping to determine which measures can be taken during the design phase to increase the robustness of an LC-VCO to high frequency substrate noise.

\section{ACKNOWLEDGMENT}

The authors would like to thank Benito González from IUMA-ULPGC for the design of the VCO core, the Centre Tecnològic de Telecomunicacions de Catalunya and Agilent Technologies and Dídac Gómez for their support during the measurements.

\section{REFERENCES}

[1] M. Mendez, D. Mateo, X. Aragones, and J. Gonzalez, "Phase noise degradation of lc-tank vcos due to substrate noise and package coupling," ESSCIRC 2005, pp. 105 - 108, sept. 2005.

[2] C. Soens, G. Van der Plas, P. Wambacq, and S. Donnay, "Performance degradation of an lc-tank vco by impact of digital switching noise," ESSCIRC 2004., pp. 119 - 122, sept. 2004.

[3] B. Razavi, "A study of phase noise in cmos oscillators," Solid-State Circuits, IEEE Journal of, vol. 31, no. 3, pp. 331 -343, mar 1996.

[4] N. Checka, D. Wentzloff, A. Chandrakasan, and R. Reif, "The effect of substrate noise on vco performance," Radio Frequency integrated Circuits (RFIC), pp. 523 - 526, june 2005.

[5] R. Adler, "A study of locking phenomena in oscillators," Proceedings of the IRE, vol. 34, no. 6, pp. $351-357$, june 1946.

[6] H. Stover, "Theoretical explanation for the output spectra of unlocked driven oscillators," Proceedings of the IEEE, vol. 54, no. 2, pp. $310-$ 311, feb. 1966.

[7] M. Armand, "On the output spectrum of unlocked driven oscillators," Proceedings of the IEEE, vol. 57, no. 5, pp. 798 - 799, may 1969.

[8] B. Razavi, "A study of injection locking and pulling in oscillators," Solid-State Circuits, IEEE Journal of, vol. 39, no. 9, pp. 1415 - 1424, sept. 2004.

[9] J. Dekleva and I. Zanchi, "Improved calculation for the output spectra of unlocked driven oscillators," Proceedings of the IEEE, vol. 60, no. 1, pp. $135-136$, jan. 1972.

[10] M. Molina, X. Aragones, and J. Gonzalez, "Experimental analysis of substrate isolation techniques for rf-soc integration," SOC Conference, 2009. IEEE International, pp. 199 -202, sept. 2009. 